\title{
Relación del feed-back y las barreras de comunicación del docente con la motivación intrínseca de estudiantes adolescentes de educación física
}

\author{
Juan Antonio Moreno-Murcia, Elisa Huéscar*, Noelia Peco, Elena Alarcón y Eduardo Cervelló
}

Universidad Miguel Hernández de Elche, España

\begin{abstract}
Resumen: Este estudio examinó la relación entre los diferentes tipos de feed-back y la evitación de las barreras de la comunicación por parte del docente con la motivación intrínseca del alumnado en clases de educación física. Los estudiantes $(n=214)$ cumplimentaron varios cuestionarios que valoraban la percepción del feed-back del docente, el uso de estrategias de comunicación para evitar las barreras de comunicación con el alumnado y la motivación intrínseca. Los resultados mostraron que los tipos de feed-back general positivo percibido, conocimiento del rendimiento percibido y no verbal positivo general correlacionaban tanto positivamente entre sí, como también con la evitación de barreras de la comunicación y la motivación intrínseca del alumnado. Además, los análisis de regresión realizados revelaron que el uso del feed-back general positivo percibido y del conocimiento del rendimiento percibido predijeron un clima de aula exento de barreras de la comunicación. Los resultados son discutidos en el marco de una mejora de la calidad de la docencia en clases de educación física.

Palabras clave: Comunicación verbal; comunicación no verbal; barreras de la comunicación; motivación intrínseca; educación física.
\end{abstract}

Title: Relations of feed-back and teacher communication barriers with intrinsic motivation among adolescent students in physical education.

Abstract: This study examined the relationship between different types of feed-back and avoiding the teacher's communication barriers with students and intrinsic motivation in physical education classes. Students $(n=214)$ completed a set of questionnaires that assessed the perception of the teacher feedback, the use of communication strategies to overcome the barriers of communication with students and intrinsic motivation. The results showed positive relationships between the general positive feedback received, the knowledge of perceived performance and general positive and nonverbal feedback, as well as the avoidance of barriers to communication and students' intrinsic motivation. In addition, regression analysis revealed that use of the generally positive feedback and the knowledge of the perceived performance predicted a classroom atmosphere free of communication barriers. The results are discussed in the context of improving the quality of teaching in physical education classes.

Key words: Verbal communication; nonverbal communication; communication barriers; intrinsic motivation; physical education.

\section{Introducción}

La investigación reciente en el ámbito del deporte y la motivación en educación física vienen destacando el importante papel del feed-back del docente sobre la calidad del proceso de enseñanza-aprendizaje (Koka y Hein, 2005; Sánchez y Viciana, 2002; Valdivia, 2011; Vernetta y López, 1998). En este sentido, se considera que un feed-back adecuado favorece el aprendizaje de los estudiantes (Pieron, 1992), así como la actuación del docente (Grant, Ballard, y Glynn, 1990; Ureña, Alarcón y Ureña, 2009). Concretamente, el aspecto que más se ha relacionado con el feed-back del docente de educación física es la motivación de su alumnado, tomando como marco teórico los supuestos básicos de la teoría de la autodeterminación (Decy y Ryan, 2000). Según esta perspectiva, el origen de la motivación puede ser más interno o más externo al practicante (más o menos autodeterminado), en la medida en que las personas se involucran o no libremente en la realización de sus actividades para conseguir ciertas metas, es decir, el grado en que realizan sus acciones de forma voluntaria, por propia elección.

De acuerdo con esta teoría, la forma más autodeterminada de motivación es la motivación intrínseca, en la que la actuación es motivada por el placer y el disfrute que genera la propia actividad. Así pues, parece que los diferentes tipos de feed-back del docente que el alumnado percibe, están influyendo en el grado de motivación del estudiante respecto a las tareas propuestas, constituyéndose de esta forma el factor

* Dirección para correspondencia [Correspondence address]: Juan Antonio Moreno-Murcia. Universidad Miguel Hernández de Elche. Centro de Investigación del Deporte. Avenida de la Universidad, s/n. 03202 Elche, Alicante (España). E-mail: j.moreno@umh.es motivacional como una de las variables mediadoras más importantes del proceso de aprendizaje. En este sentido, los estudios muestran una relación positiva entre el feed-back sobre información de carácter positivo y altos niveles de motivación intrínseca y percepción de competencia por parte del alumnado de educación física (Amorose y Smith, 2003; Koka y Hein, 2003; Nicaise, Cogerino, Bois, y Amorose, 2006; Reinboth, Duda, y Ntoumanis, 2004). Por su parte, el feedback de carácter negativo se ha relacionado de manera inversa (negativa), con la motivación intrínseca del alumnado (Hollembeak y Amorose, 2005; Vallerand y Reid, 1984; Whitehead y Corbin, 1991). Sin embargo, un aspecto del feed-back al que se le ha prestado muy poca atención en la investigación hasta el momento y que resulta de esencial importancia dentro del proceso de comunicación del docente con su alumnado es el que se refiere a los aspectos no verbales del mensaje que perciben los estudiantes y sus efectos sobre la motivación. En este sentido, y pese a que la importancia del mensaje no verbal se viene destacando desde los años cincuenta (Birdwhistell, 1952), en la actualidad son muy escasos los estudios en el ámbito de la educación física que focalizan sobre el papel del feed-back no verbal del docente percibido por el alumnado en clases de educación física (Koka y Hein, 2005; Vallejo, 2007, 2008; Vallejo y Plested, 2008). Además, a pesar de los abundantes hallazgos respecto a los diferentes tipos de feed-back verbal del docente (Amorose y Horn, 2000), en algunos estudios que además han contemplado el papel del feed-back no verbal del docente sobre la motivación intrínseca del alumnado, las relaciones con la motivación intrínseca del alumnado no se han podido demostrar (Koka y Hein, 2005).

Por otro lado, dentro del proceso de comunicación, existen una serie de factores, obstáculos o interferencias que la 
literatura especializada ha llamado "barreras de la comunicación" y que la investigación de los últimos años señala su importancia en la fluidez, estilo y comprensión del mensaje que transmite el docente a su alumnado (Arellano, 2006). Las barreras de la comunicación pueden limitar la exactitud del mensaje que el docente de educación física trasmite a su alumnado, por lo que el docente debería asegurarse no solamente de que el mensaje sea recibido por el estudiante, sino que además, sea decodificado y entendido correctamente. En este sentido, la habilidad para una escucha activa parece ser el elemento potencialmente distorsionador más destacado por los autores (Echeverría, 2003; Elizondo, 2004; Marroquín, 1984). Este aspecto hace referencia a la forma de escuchar con atención lo que dice el estudiante, tanto en su mensaje verbal como no verbal. Así pues, este ingrediente, junto a una actitud del docente de respeto, cordialidad, empatía e interés hacia el estudiante, son factores que podrían favorecer el acercamiento e interés del alumnado hacia el contenido.

Otro aspecto importante en el análisis del feed-back en educación física tiene que ver con el sexo del alumnado. En este sentido, una amplia línea de investigación demuestra que el docente de educación física suele otorgar, en ocasiones de manera involuntaria, un trato desigual a su alumnado en función del sexo (Del Villar, 1996; Duffy, Warren, y Walsh, 2001; Griffin, 1989; Hastie y Pickwell, 1996). Daniels (1983) reflejó que los chicos pensaban que sus docentes les elogiaban más y eran más atentos con ellos que con las chicas. Concretamente, la investigación señala que existe una mayor aportación de feed-back a los chicos que a las chicas (Davis, 2003; Jiménez, Ramos, y Cervelló, 2002; Nicaise, Cogerino, Fairclough, Bois, y Davis, 2007), sobre todo del tipo de conocimento del rendimiento (Subirats, 2007), organizativo o por mala conducta (Nicaise et al., 2007). Estos aspectos podrían estar influyendo en la auto-percepción del alumnado y disminuir por ende su nivel de autoconfianza y de motivación respecto al aprendizaje (Martinek, 1989).

En definitiva, a partir de estudios previos (Cervelló y Santos-Rosa, 2000) que consideran que las diferencias individuales en las capacidades comunicativas del docente (verbales y no verbales), así como en la creación de un clima de aprendizaje estimulante (minimizando las barreras de comunicación), pueden tener una repercusión sobre la motivación del alumnado en educación física. Y, en segundo lugar, dada esta descompensación en la investigación sobre el papel diferencial de los distintos tipos de feed-back del docente en clases de educación física sobre la motivación intrínseca del alumnado y la escasa investigación sobre las barreras de la comunicación en educación física, este trabajo se diseñó para responder a una serie de interrogantes concretos. En primer lugar, se investigaron las relaciones entre los diferentes tipos de feed-back, la evitación de barreras de la comunicación y la motivación intrínseca, con el fin de comprobar si existían determinados tipos de feed-back, tanto de carácter verbal como no verbal, que pudieran relacionarse con la motivación intrínseca del alumnado en clases de educación física. En concreto, hipotetizamos que los tipos de feed-back positivo general, de conocimiento del rendimiento percibido y no verbal positivo, predecirían la motivación intrínseca del alumnado en clases de educación física. Además, en la línea de los estudios que recomiendan al docente el uso equilibrado de ambos tipos de comunicación, verbal y no verbal, para la consecución de un mensaje coherente y consistente ( $\mathrm{Yu}$ kelson, 1998), esperábamos que el feed-back positivo general y el feed-back no verbal positivo correlacionaran positivamente entre sí. Segundo, esperábamos que el feed-back no verbal negativo general, se relacionara de manera negativa con la motivación intrínseca del estudiante. Tercero, se hipotetizó que el uso de estrategias para evitar las barreras de la comunicación por parte del docente, se relacionaría positivamente con el feed-back de carácter positivo (feed-back positivo general y feed-back no verbal positivo) y con el conocimiento del rendimiento percibido y que además tendría un efecto positivo sobre la motivación intrínseca del alumnado en clases de educación física. A continuación, nos interesó comprobar la predicción tanto del uso de estrategias para evitar las barreras de la comunicación como de la motivación intrínseca respecto a los tipos de feed-back considerados, esperando que ambas variables predigan positivamente la utilización de los tipos de feed-back de carácter positivo para el alumnado. Por último, debido a que, como hemos señalado más arriba, la investigación anterior sobre el tema (Nicaise et al., 2006) viene mostrando que los efectos del feed-back del docente son más fuertes para los chicos que para las chicas, también analizamos la posible relación diferencial del sexo respecto a las variables de nuestro estudio.

\section{Método}

\section{Participantes}

La muestra ha estado compuesta por 214 estudiantes (113 chicos y 101 chicas) adolescentes de seis centros públicos de Enseñanza Secundaria Obligatoria de la provincia de Alicante en clases de educación física, con una edad media de 14.0 años $(D T=1.21)$.

\section{Instrumentos}

Cuestionario de las percepciones del feed-back del docente-revisado (PTF-R). Se utilizó la versión completa revisada y ampliada (Koka y Hein, 2005) de la Perceptions of Teacher's Feedback scale (Koka y Hein, 2003) validada al contexto español por Moreno-Murcia y Huéscar (2012). Esta escala está compuesta por un total de 14 ítems agrupados en cuatro factores, los dos primeros de carácter verbal y los dos siguientes de carácter no verbal: feed-back general positivo percibido (tres ítems) (e.g. "Mi trabajo es con frecuencia animado/alentado por el docente"), conocimiento del rendimiento (ejecución) percibido (cuatro ítems) (e.g. "A menudo el docente me da instrucciones durante la ejecución”), feed-back no verbal negativo percibido (tres ítems) (e.g. "El docente mueve su cabeza 
cuando realizo una mala ejecución) y feed-back no verbal positivo percibido (cuatro ítems) (e.g. "El docente me elogia a pesar de que no lo merezca"). Estos ítems estaban encabezados por la frase "En mis clases de educación física...", que se respondían con una escala Likert de 1 (Falso) a 5 (Verdadero). La consistencia interna reveló un valor alfa de Cronbach de $.75, .68, .69$ y .72 , respectivamente.

Escala de evitación de barreras de comunicación en educación física (EEBCEF). Se utilizó la escala de Moreno-Murcia, Huéscar, Peco, Alarcón, y Cervelló (en prensa) para medir la creación de un clima de aprendizaje que minimizara las barreras de comunicación en educación física. A esta escala, le encabezaba el enunciado "El docente en clases de educación física...". Está compuesta de 12 ítems (e.g. "No me interrumpe cuando hablo con él"), que se responden en una escala tipo Likert que oscila entre 1 (Nunca) y 5 (Siempre). La consistencia interna de la dimensión fue de .79 .

Motivación intrínseca. Se empleó el factor motivación intrínseca de la Escala del Locus Percibido De Causalidad (PLOC Scale) de Goudas, Biddle, y Fox (1994), validada al contexto español por Moreno, González-Cutre, y Chillón (2009). Este factor, encabezado por el enunciado "Participo en esta clase de educación física...", está compuesto por cuatro ítems (e.g. "Porque disfruto aprendiendo nuevas habilidades") que se responden con una escala tipo Likert que va de 1 (Totalmente en desacuerdo) a 7 (Totalmente de acuerdo). La consistencia interna reveló un valor alfa de Cronbach de .84 .

\section{Procedimiento}

Para la recogida de la información nos pusimos en contacto con 12 Centros de Enseñanza Secundaria Obligatoria, informándoles de los objetivos del estudio y solicitando colaboración en el mismo. Una vez se obtuvo la correspondiente autorización de los padres de los estudiantes, se realizó la administración de los cuestionarios bajo la supervisión del investigador principal del estudio. El tiempo requerido para cumplimentar los cuestionarios fue de 12 minutos, aproximadamente. El carácter de realización del estudio fue anónimo y la participación del alumnado fue voluntaria.

\section{Análisis de los datos}

Se obtuvieron los estadísticos descriptivos de las diferentes variables de estudio y se realizó el análisis de consistencia interna de los factores a través del alfa de Cronbach. Posteriormente se aplicó un análisis de correlación de Pearson entre todas las variables del estudio. También se realizaron análisis de regresión lineal hacia delante por pasos para analizar la predicción de la motivación intrínseca a través del feed-back y para analizar la predicción de las barreras de comunicación a través del feed-back. Por último, se llevó a cabo un Análisis de diferencia de medias para muestras independientes por sexo. Estos análisis se desarrollaron con el paquete estadístico SPSS 18.0.

\section{Resultados}

\section{Análisis descriptivo y de correlación de todas las varia- bles}

En la Tabla 1, se presentan los estadísticos descriptivos de todas las variables del estudio así como las correlaciones bivariadas, respectivamente. Como se puede observar, los estudiantes asignaron una mayor puntuación al conocimiento del rendimiento percibido, seguido del feed-back general positivo percibido y del feed-back no verbal positivo general. El feed-back no verbal negativo general fue el que obtuvo la menor puntuación. La evitación de las barreras de la comunicación presentó una puntuación media de 4.18 y la motivación íntrínseca de 5.70. Tal y como se plantea en el primer objetivo del estudio, en el análisis de correlación resultó que los tipos de feed-back general positivo percibido, conocimiento del rendimiento percibido y no verbal positivo general, correlacionaban entre sí positiva y significativamente, $y$, además, se relacionan de forma positiva y significativa con la motivación intrínseca del alumnado.

Tabla 1. Media $(M)$, Desviación Típica $(D T)$, Coeficiente Alfa $(\alpha)$ y Análisis de Correlación de Todas las Variables.

1. Feed-back general positivo percibido

2. Conocimiento del rendimiento percibido

3. Feed-back no verbal negativo general

4. Feed-back no verbal positivo general

5. Barreras de comunicación

6. Motivación intrínseca

\begin{tabular}{lllllllll}
$M$ & $D T$ & $\alpha$ & 1 & 2 & 3 & 4 & 5 & 6 \\
\hline 3.56 & .92 & .75 & - & $.54^{* *}$ & .00 & $.61^{* *}$ & $.66^{* *}$ & $.40^{* *}$ \\
3.86 & .74 & .68 & - & - & .07 & $.44^{* *}$ & $.51^{* *}$ & $.24^{* *}$ \\
2.47 & .98 & .69 & - & - & - & $.17^{* *}$ & -.11 & -.09 \\
2.78 & .93 & .72 & - & - & - & - & $.44^{* *}$ & $.34^{* *}$ \\
4.18 & .69 & .79 & - & - & - & - & - & $.42^{* *}$ \\
5.70 & 1.30 & .84 & - & - & - & - & - & - \\
\hline
\end{tabular}

$* * p<.001$

En segundo lugar, el feed-back no verbal negativo general no mostró relación alguna con la motivación intrínseca, relacionándose positiva y significativamente con el feed-back no verbal positivo general. Por último, la evitación de las barreras de la comunicación correlacionó significativa y positivamente con los tipos de feed-back positivo general, conocimiento del rendimiento y no verbal positivo general, así co- mo también se relacionó de la misma forma con la motivación intrínseca del alumnado.

\section{Análisis de regresión lineal}

Con el objetivo de comprobar el valor predictivo de la variable evitación de las barreras de la comunicación por 
parte del docente sobre los cuatro tipos de feed-back percibidos por el alumnado en clases de educación física, se realizó un análisis de regresión lineal hacia delante por pasos (Tabla 2), siendo la variable dependiente la evitación de las barreras de la comunicación y las variables independientes, los cuatro tipos de feed-back del docente (positivo general, conocimiento del rendimiento, no verbal negativo y no verbal positivo). Los resultados mostraron que tres de las cuatro dimensiones del feed-back del docente (el feed-back general positivo percibido y el conocimiento del rendimiento percibido de manera positiva y significativa, y el feed-back no verbal negativo general de forma negativa y significativa) predecían de manera significativa la evitación de las barreras de la comunicación. La varianza explicada obtenida fue del $48 \%$.

Tabla 2. Análisis de Regresión Lineal Para la Predicción de las Barreras de Comunicación.

\begin{tabular}{lllll}
\hline & $\mathrm{B}$ & $\mathrm{SEB}$ & $\beta$ & $\Delta R^{2}$ \\
\hline & 2.14 & .20 & & $.48^{* *}$ \\
Feed-back general positivo percibido & .36 & .05 & $.49^{* *}$ & \\
Conocimiento del rendimiento percibi-.21 & .05 & $.23^{* *}$ & \\
do & & & & \\
Feed-back no verbal negativo general & -.10 & .03 & $-.14^{*}$ & \\
Feed-back no verbal positivo general & .05 & .04 & .06 & \\
\hline$* p<.01 ; * p<.001$ & & &
\end{tabular}

$* p<.01 ; * * p<.001$

Este mismo proceso se repitió respecto a la motivación intrínseca de los estudiantes. Así pues, para comprobar el poder de predicción de esta variable sobre los cuatro tipos de feed-back del alumnado se realizó el correspondiente análisis de regresión hacia delante por pasos (Tabla 3). En este caso, los resultados mostraron que la motivación intrínseca predecía de manera significativa y positiva el feed-back general positivo percibido y el feed-back no verbal positivo general, y de forma significativa pero negativa, el feed-back no verbal negativo general. La varianza explicada obtenida fue del $17 \%$.

Tabla 3. Análisis de Regresión Lineal Para la Predicción de la Motivación Intrínseca.

\begin{tabular}{lllll}
\hline & $\mathrm{B}$ & $\mathrm{SEB}$ & $\beta$ & $\Delta \mathrm{R}^{2}$ \\
\hline & 3.85 & .47 & & $.17^{* *}$ \\
Feed-back general positivo percibido & .37 & .12 & $.26^{*}$ & \\
Conocimiento del rendimiento perci-.03 & .13 & .02 & \\
bido & & & & \\
Feed-back no verbal negativo general & -.16 & .08 & $-.12^{*}$ & \\
Feed-back no verbal positivo general & .27 & .11 & $.19^{*}$ & \\
$*_{p<.01 ; *}^{* *}<.001$ & & & &
\end{tabular}

\section{Análisis de diferencia de medias para muestras inde- pendientes por sexo}

Para observar las posibles diferencias por sexo respecto a los cuatro tipos de feed-back del docente que el alumnado percibía con la evitación de las barreras de la comunicación del docente, se realizó un análisis de diferencia de medias para muestras independientes por sexo (Tabla 4). Tras el test de Levene se comprobó la homogeneidad de las varianzas, por lo que después del análisis de las diferencias de medias solamente se encontraron diferencias significativas en el caso de los chicos para el feed-back no verbal positivo general $(p<$ $.01)$.

Tabla 4. Análisis de Diferencia de Medias para Muestras Independientes por Sexo.

\begin{tabular}{llllll} 
& Sexo & $M$ & $D T$ & $t$ & $p$ \\
\hline Feed-back general positivo per- & Chico & 3.67 & .87 & 1.82 & .070 \\
cibido & Chica & 3.44 & .96 & & \\
Conocimiento del rendimien- & Chico & 3.80 & .77 & -1.26 & .208 \\
to percibido & Chica & 3.93 & .70 & & \\
Feed-back no verbal negativo & Chico & 2.42 & 1.00 & -.86 & .390 \\
general & Chica & 2.53 & .96 & & \\
Feed-back no verbal positivo & Chico & 2.95 & .95 & 2.89 & .004 \\
general & Chica & 2.58 & .87 & & \\
Barreras de comunicación & Chico & 4.18 & .62 & .15 & .879 \\
& Chica & 4.17 & .76 & & \\
\hline
\end{tabular}

\section{Discusión}

Desde hace ya dos décadas en el ámbito de la educación física se viene subrayando la relación positiva de un clima de clase de carácter participativo y positivo y la motivación intrínseca del alumnado (e.g. Biddle, Akande, Vlachopoulos, y Fox 1996; Goudas y Biddle, 1994; Papaioannou, 1994). Además, desde entonces, muchos estudios han demostrado el potencial impacto de un clima de clase motivante sobre resultados óptimos tanto psicológicos como sociales para el alumnado (Lloyd y Fox, 1992). Sin embargo, y pese a que la actuación del docente sobre la motivación intrínseca del estudiante de educación física se ha señalado como fundamental para conseguir aumentar o disminuir la motivación del alumnado (Goudas et al., 1995), en la actualidad, todavía existe una falta de concreción sobre qué actuaciones concretas del docente, tanto de carácter verbal como no verbal, son las que están influyendo, tanto en sentido positivo, como negativo, en el grado de motivación del alumnado en clases de educación física. En este sentido, el objetivo de este estudio fue analizar la relación entre la percepción de cuatro tipos de feed-back del docente y de diferentes estrategias para evitar las barreras de la comunicación en clases de educación física con la motivación intrínseca del alumnado. Pese a que los diferentes tipos de feed-back han sido relacionados con frecuencia en los trabajos con las consecuencias motivacionales que producen (Amorose y Horn, 2000; Amorose y Smith, 2003; Fredenburg, Lee, y Solomon, 2001; Koka y Hein, 2003) son escasos los estudios que analizan la relación existente entre el tipo de feed-back del docente y las barreras de la comunicación en educación física con la motivación intrínseca del estudiante en educación física. Por lo tanto, este estudio da un paso más y añade al análisis del tipo de feedback del docente percibido por el alumnado, el papel del uso de estrategias para evitar las barreras de la comunicación, para relacionarlos con la motivación intrínseca del discente de educación física. 
Así pues, el análisis de correlaciones mostró que los tipos de feed-back general positivo percibido, conocimiento del rendimiento percibido y no verbal positivo general, correlacionaban entre sí positiva y significativamente. En este sentido, el trabajo efectuado por Koka y Hein (2003), demostró también, en un estudio correlacional, la existencia de relaciones positivas entre la percepción del feed-back positivo general y el feed-back de conocimiento del rendimiento en clases de educación física. Respecto al feed-back no verbal positivo general, nuestros resultados confirman una relación positiva y significativa con los dos tipos de feed-back que contienen información de carácter verbal, esto es, con el feed-back general positivo percibido y con el conocimiento del rendimiento percibido. Este hallazgo está en consonancia con la sugerencia que hacen algunos autores de que debería existir una coherencia en la transmisión del contenido verbal y no verbal del mensaje por parte del docente hacia su alumnado (e.g. Yukelson, 1998).

Por otro lado, nuestro estudio apoya y corrobora la amplia línea de investigación que relaciona positivamente el feedback de carácter positivo con la motivación intrínseca del alumnado de educación física (Allen y Howe, 1998; Amorose y Horn, 2000; Amorose y Smith, 2003; Fredenburg et al., 2001). En este sentido, nuestros datos mostraron que el feedback general positivo percibido y el feed-back no verbal positivo general predecían positivamente la motivación intrínseca del alumnado, mientras que el feed-back no verbal negativo general, lo hacía en sentido negativo. En la actualidad, y pese a que los estudios vienen subrayando la importancia del mensaje no verbal desde hace años (e.g. Birswhistell, 1952), son escasas las investigaciones en el ámbito educativodeportivo que contemplan la variable no verbal en sus modelos. Por ejemplo, Koka y Hein (2005) en un estudio con estudiantes de educación física, hipotetizaron que el feed-back no verbal del docente se relacionaría con la motivación intrínseca del alumnado, pero los resultados no confirmaron su hipótesis. Según nuestros datos, es posible que el hecho de dividir el feed-back no verbal según su naturaleza positiva o negativa, nos ayude a clarificar estas relaciones. En este sentido, hemos encontrado, en la línea de la investigación sobre el tema, que el feed-back no verbal sobre información positiva sí predecía la motivación intrínseca del alumnado de educación física, mientras que el feed-back no verbal negativo lo hacía en sentido negativo. Posiblemente pues, el aspecto de mayor importancia para un aumento de la motivación intrínseca del alumnado sea el carácter positivo del feed-back, tanto el trasmitido de manera verbal como no verbal.

Por otro lado, como plateábamos en las hipótesis de partida, un aspecto de especial interés de este estudio fue la inclusión de la variable sobre el uso de estrategias para evitar las barreras de la comunicación por parte del docente de educación física. En este sentido, nuestros resultados mostraron que tanto el feed-back general positivo percibido, como el conocimiento del rendimiento percibido predecían positivamente el uso de estrategias para evitar las barreras de la comunicación en clases de educación física. En consonancia, la percepción del feed-back no verbal negativo general, predecía negativamente la evitación de las barreras de la comunicación. Esto significaría que la inclusión en la práctica docente habitual del refuerzo positivo, concretamente, del feedback positivo general y la información sobre el rendimiento de su alumnado, podría favorecer además un clima de clase definido por un trato cordial y amable con el alumnado y una actitud de respeto, de empatía y de cercanía. Además, estos aspectos, definidos en nuestro trabajo por la variable evitación de las barreras de la comunicación del docente, se relacionaron en nuestro estudio de manera significativa y positiva con la motivación intrínseca del alumnado en clases de educación física, por lo que podemos sugerir que, además de la práctica de un feed-back que contenga información de carácter positivo, tanto de forma verbal como no verbal, para mejorar la motivación intrínseca del alumnado de educación física, también sería recomendable la creación de un clima de clase exento de barreras de la comunicación. Una implicación pedagógica derivada de estos resultados podría ser por ejemplo, la necesidad del docente de ser consciente de que probablemente, una mayor utilización del feed-back de carácter positivo con su alumnado, así como cuidar otros aspectos menos evidentes como la creación de un clima de confianza y libertad de expresión en el que el alumnado se sienta cómodo, podría repercutir en un mayor número de experiencias de clase positivas y obtener como resultado un clima de clase más motivante.

También nos interesó comprobar si existían diferencias por sexo para la percepción tanto de los diferentes tipos de feed-back como de la evitación de las barreras de la comunicación del docente en clases de educación física. Los resultados mostraron que solamente existían diferencias significativas a favor de los chicos para la variable feed-back no verbal positivo general. Numerosos estudios observacionales vienen encontrando que, en general, los chicos reciben más atención, feed-back e información técnica que las chicas (Drudy y Úi Chatháin, 2002; Nicaise et al., 2006). En concreto, nuestro hallazgo se une a la amplia línea de investigación que afirma que los chicos se benefician en mayor medida que las chicas del feed-back de carácter positivo (Deci, 1972; Deci, Cascio, y Krusell, 1975; Dunbar y O’Sullivan, 1986; Nicaise et al., 2006). Algunos trabajos han atribuido este resultado al hecho de que parece ser que los chicos inician un mayor número de interacciones con sus docentes que las chicas, por lo que aumentan la probabilidad de obtener mayor feedback del docente (Altermatt, Jovanovic, y Perry, 1998).

En conclusión, los resultados de este estudio apoyan la idea de que la motivación intrínseca en clases de educación física se ve afectada por el papel del feed-back del docente, concretamente del feed-back de carácter positivo. En definitiva, nuestros resultados son acordes con los hallados por Koka y Hein (2003) y Nicaise et al. (2006) respecto a las orientaciones comunicativas que debería seguir el docente de educación física para incentivar la motivación intrínseca de su alumnado, aunque este estudio añade el hallazgo de la relación positiva y significativa del uso de las estrategias para 
evitar las barreras de la comunicación y la motivación intrínseca del estudiante en clases de educación física. Por otro lado, la toma de conciencia por parte del docente sobre un trato igualitario al alumnado independientemente del sexo debería ser un objetivo a buscar en las clases de educación física. Probablemente, la sensación de seguridad proporcionada por el aumento del uso de estrategias comunicativas del docente encaminadas a fomentar la percepción de autoeficacia en las chicas, podría repercutir en un mayor número de interacciones docente-alumna y por ende, en un aumento del feed-back percibido en el grupo de las chicas.

Posteriores trabajos, deberán seguir profundizando, desde una perspectiva experimental, en los efectos que la percepción de diferentes tipos de feed-back tienen sobre la motivación intrínseca del alumnado en clases de educación física. De manera más concreta, se deberá confirmar el papel de las

\section{Referencias}

Allen, J. B., y Howe, B. L. (1998). Player ability, coach feedback, and female adolescent athletes' perceived competence and satisfaction. Journal of Sport \&o Exercise Psychology, 20, 280-299.

Altermatt, E. R., Jovanovic, J., y Perry, M. (1998). Bias or reponsivity? Sex and achievement-level effects on teachers' classroom questioning practices. Journal of Educational Psychology, 90, 516-527.

Amorose, A. J., y Horn, T. S. (2000). Intrinsic motivation: relationships with collegiate athletes' gender, scholarship status, and perceptions of their coaches' behavior. Journal of Sport and Exercise Psychology, 22, 63-84.

Amorose, A. J., y Smith, P. (2003). Feedback as a source of physical competence information: Effects of age, experience and type of feedback. Journal of Sport and Exercise Psychology, 25, 341-359.

Arellano, N. (2006). Las barreras en la comunicación no verbal entre docente-alumno. Revista Cientifica de Ciencias Humanas, 2(4), 3-38.

Biddle, S., Akande, A., Vlachopoulos, S., y Fox, K. R. (1996). Towards an understying of children's motivation for physical activity: Achievement goal orientations, beliefs about sport success, and sport emotion in Zimbabwean children. Psychology and Health, 12, 49-55.

Birdwhistell, R. L. (1952). Introduction to Kinesics: Annotated System for Analisis of Body Motion and Gesture. Washington, D.C.; Department of State, Foreign Service Institute.

Cervelló, E. M., y Santos-Rosa, F. J. (2000). Motivación en las clases de educación física: un estudio de la perspectiva de las metas de logro en el contexto educativo. Revista de Psicología del Deporte, 9, 51-70.

Daniels, R. (1983). The development of a tool to measure perceptions of physical education teaching bejaviors found in secondary schools. Unpublished doctoral dissertation. University of North Carolina, Greensboro.

Davis, K. L. (2003). Teaching for gender equity in physical education: A review of literature. Women in Sport and Physical Activity Journal, 12, 55-82.

Deci, E. L., y Ryan, R. M. (2000). The "what" and "why" of goal pursuits: Human needs and the self-determination of behaviour. Psychological Inquiry, 11, 227-268.

Del Villar, F. (1996). La evolución del pensamiento docente durante las prácticas didácticas. Un estudio de casos en Educación Física. En F. Del Villar (Ed.), La investigación en la enseñanza de la Educación Física (pp. 203-231). Cáceres: Servicio de Publicaciones. Universidad de Extremadura.

Drudy, S., y Úi Chatháin, M. (2002). Gender effects in classroom interaction: Data collection, self-analysis, and reflection. Evaluation and Research in Education, 16, 34-50.

Duffy, J., Warren, K., y Walsh, M. (2001). Classroom interactions: Gender of teacher, gender of student, and classroom subject. Sex Roles, 45, 579593.

Dunbar, R., y O'Sullivan, M. (1986). Effects of intervention on differential treatment of boys and girls in elementary physical education lessons. Journal of Teaching in Physical Education, 5, 166-175. barreras de la comunicación sobre el entramado de la motivación, especificando qué comportamientos debe adquirir el docente en clases de educación física para mejorar la implicación del alumnado y optimizar así la calidad del proceso de enseñanza-aprendizaje. Todo ello sin olvidar la necesidad de seguir explorando las razones de la percepción diferencial según el sexo del alumnado del feed-back del docente. Futuros trabajos, deberían considerar otras variables mediadoras de este proceso como el posible efecto del sexo del docente, las iniciaciones de interacción por parte del alumnado hacia el docente o la existencia de pensamientos estereotipados. Por tanto, es necesaria más investigación de corte experimental que pueda corroborar los resultados de naturaleza correlacional de este trabajo y ayude a clarificar las relaciones entre el patrón de comunicación del docente y la motivación intrínseca del alumnado en clases de educación física.

Echeverría, R. (2003). Ontología del lenguaje. Chile: J. C. Sáez.

Elizondo, M. (2004). Asertividad y escucha activa en el ámbito académico. Sevilla: Trillas.

Fredenburg, K. B., Lee, A. M., y Solomon, M. (2001). The effects of augmented feedback on students' perception and performance. Research Quarterly for Exercise and Sport, 72, 232-242.

Goudas, M., y Biddle, S. (1994). Perceived motivational climate and intrinsic motivation in school physical education classes. European Journal of Psychology of Education, 9, 241-250.

Goudas, M., Biddle, S. J. H., y Fox, K. (1994). Perceived locus of causality, goal orientations and perceived competence in school physical education classes. British Journal of Educational Psychology, 64, 453-463.

Goudas, M., Biddle, S .J. H., Fox, K. R., y Underwood, M. (1995). It ain't what you do, it's the way that you do it! Teaching style affects children motivation in track and field lessons. The Sport Psychologist, 9, 254-264

Grant, B., Ballard, K., y Glynn, T. (1990). Teacher feedback intervention, motor on task behavior, and successful task performance. Journal of Teaching in Physical Education, 9, 123-139.

Griffin, P. S. (1989). Gender as a socializing agent in Physical Education. En T. J. Templin y P. G. Schempp (Eds.), Learning to teach: Socialization into Physical Education (pp. 219-233). Indianapolis, IN: Benchmark Press.

Hastie, P. A., y Pickwell, A. (1996). Take your partners: A description of a student social system ina secondary school dance class. Journal of Teaching in Physical Education, 15, 171-187.

Hollembeak, J., y Amorose, A. (2005). Perceived coaching behaviors and college athletes' intrinsic motivation: a test of self-determination theory. Journal of Applied Sport Psychology, 17(1), 20-36.

Jiménez, R., Ramos, L. A., y Cervelló, E. (2002). Análisis de la coeducación en las clases de Educación Física. Propuestas didácticas para una intervención no sexista en el contexto educativo. Habilidad Motriz, 18, 39-47.

Koka, A., y Hein, V. (2003). Perceptions of teacher's feedback and learning environment as predictors of intrinsic motivation in physical education. Psychology of Sport and Exercise, 4, 333-346.

Koka, A., y Hein, V. (2005). The effect of perceived teacher feed-back on intrinsic motivation in physical education. International Journal of Sport Psychology, 36(2), 91-106.

Lloyd, J., y Fox, K. R. (1992). Achievement goals and motivation to exercise in adolescent girls: A preliminary intervention study. British Journal of Physical Education Research Supplement, 2, 12-16.

Marroquin, M. (1984). La escucha activa. Madrid: Asetes.

Martinek, T. J. (1989). Children's perceptions of teaching behaviors: an attributional model for explaining teacher expectancy effects. Journal of Teaching in Physical Education, 8, 318-328.

Moreno, J. A., González-Cutre, D., y Chillón, M. (2009). Preliminary validation in Spanish of a scale designed to measure motivation in physical 
education classes: the Perceived Locus of Causality (PLOC) Scale. Spanish Journal of Psychology, 12, 327-337.

Moreno-Murcia, J. A., y Huéscar, E. (2012). Relación del tipo de feed-back del docente con la percepción de autonomía del alumnado en clases de educación física. Infancia y Aprendizaje, 35(1), 87-98.

Moreno-Murcia, J. A., Huéscar, E., Peco, N., Alarcón, E., y Cervelló, E. (en prensa). Relación de la comunicación del docente con la motivación intrínseca de estudiantes adolescentes en clases de educación física. Universitas Psychologica.

Nicaise, V., Cogerino, G., Bois, J., y Amorose, A. J. (2006). Students' perceptions of teacher feedback and physical competence in physical education classes: Gender effects. Journal of Teacbing in Physical Education, 25(1), 36-57.

Nicaise, V., Cogerino, G., Fairclough, S. J., Bois, J., y Davis, K. L. (2007). Teacher feedback and interactions in physical education: Effects of student gender and physical activities. European Physical Education Review, 13(3), 319-337.

Piéron, M. (1992). La investigación en la enseñanza de las actividades físicas y deportivas. Apunts, 30, 6-19.

Reinboth, M., Duda, J. L., y Ntoumanis, N. (2004). Dimensions of coaching behavior, need, satisfaction, and the psychological and physical welfare of young athletes. Motivation and Emotion, 28(3), 297-313.

Sánchez, D. L., y Viciana, J. (2002). Análisis del discurso de un entrenador de fútbol. Comparación entre dos situaciones diferentes de competición. Motricidad, 8, 161-174.

Subirats, M. (2007). Balones fuera. Reconstruir los espacios desde la coeducación. Barcelona: Octaedro.
Ureña, N., Alarcón, F., y Ureña, F. (2009). La realidad de los deportes colectivos en la Enseñanza Secundaria. Cómo planifican e intervienen los profesores de Murcia. Retos, nuevas tendencias en Educación Física, Deportes y Recreación, 16, 9-15.

Valdivia, J. (2011). La retroalimentación correctiva y de reforzamiento en clases de educación física. Revista de Transmisión del Conocimiento Educativo $y$ de la Salud, 3(5), 687-694.

Vallejo, G. (2007). Terminologia no verbal en el deporte y la danza. Investigación adscrita al Instituto de Educación Física, Universidad de Antioquia.

Vallejo, G. (2008), La comunicación no verbal en el fútbol, la gimnasia y la danza. Medellín: J. P. Producción.

Vallejo, G., y Plested, M.C. (2008). Tipos de comunicación en interacciones deportivas. Ikala. Revista de Lenguaje y Cultura, 13(19), 1-13.

Vallerand, R. J., y Reid, G. (1984). On the causal effects of perceived competence on intrinsic motivation: A test of cognitive evaluation theory. Journal of Sport Psychology, 6, 94-102.

Vernetta, M., y López, B. J. (1998). Análisis de diferentes categorías de feedback en dos formas organizativas del medio gimnástico. Motricidad, 4, 113-130.

Whitehead, J. R., y Corbin, C. B. (1991). Youth fitness testing: The effect of percentilebased evaluative feedback on intrinsic motivation. Research Quarterly for Exercise and Sport, 62, 225-231.

Yukelson, D. (1998). Communication effectively. En J. M. Williams (Ed.), Applied sport psychology: Personal growth to peak. performance ( $3^{\text {rd }}$ ed., pp. 142157). Mountain View, CA: Mayfield.

(Artículo recibido: 03-06-2010, revisado: 16-11-2011, aceptado: 9-1-2012) 